\title{
Comprehensive Analyses of Arrhythmogenic Substrates and Vulnerability to Ventricular Tachycardia in Left Ventricular Hypertrophy in Salt-Sensitive Hypertensive Rats
}

\author{
Kenichi Kamei, MD; Kazuhira Maehara, MD; Junko Kimura, MD*; \\ Toshiyuki Ishibashi, MD; Yukio Maruyama, MD
}

\begin{abstract}
Background The contribution of conduction disturbances to susceptibility to ventricular tachycardia (VT) has not been directly examined in the process of left ventricular hypertrophy (LVH).

Methods and Results Dahl salt-sensitive (S) and -resistant rats were raised on a high-salt diet. After echocardiography, a fractionation study was conducted using wavelet transform analysis, which reflects conduction disturbances, recording of monophasic action potential (MAP), measurement of conduction velocity, and programmed extrastimuli for the induction of VT, as well as patch-clamp analysis at 10, 13 and 16 weeks (W) $(\mathrm{n}=7$, each). Systolic blood pressure, wall thickness and MAP duration significantly increased at $10 \mathrm{~W}$ in $\mathrm{S}$ rats, whereas conduction velocity decreased and MAP duration and the power of wavelet transform increased at 13W and 16W. Paired extrastimuli induced polymorphic VT only at 13W and 16W in S rats. VT frequency correlated inversely with conduction velocity and positively with MAP duration. Power of wavelet transform correlated negatively with conduction velocity and positively with VT vulnerability. The patch-clamp study revealed that the action potential duration significantly increased in $\mathrm{S}$ rats with aging, and correlated with MAP duration.

Conclusions Latently progressing slow and inhomogeneous conduction, as well as a repolarization abnormality, may contribute to VT vulnerability in hypertensive LVH. (Circ J 2007; 71: 390-396)
\end{abstract}

Key Words: Hypertrophy; Ventricular tachycardia; Wavelet analysis

$\mathbf{H}$ ypertensive left ventricular hypertrophy $(\mathrm{LVH})$ is associated with an enhanced incidence of ventricular arrhythmia and an increased risk of sudden cardiac death ${ }^{1-3}$ Although in vitro studies of hypetensive LVH have proposed various electrophysiological abnormalities (ie, impulse formation ${ }^{4,5}$ and conduction,-8 and repolarization abnormalities ${ }^{9}$ ) as arrhythmogenic substrates, it remains unclear how these factors directly relate to the vulnerability to ventricular tachyarrhythmia in the in vivo whole heart. In other words, it remains poorly understood just how these electrophysiological abnormalities develop and increase the vulnerability to ventricular tachyarrhythmia with progression of LVH. The arrhythmogenic potential in hypertensive LVH may be multifactorial, but susceptibility to ventricular tachycardia (VT) induction has not been simultaneously investigated in the same $\mathrm{LVH}$ preparations, and therefore the relationships between various underlying arrhythmogenic mechanisms and VT attack have not been clarified. A clear understanding of this pathophysiological linkage is essential for determining the therapeutic target for prevention of fatal ventricular tachyarrhythmia. To investigate this issue, we used Dahl salt-sensitive hypertensive rats in which concentric LVH develops in a relatively short

(Received August 23, 2006; revised manuscript received November 22, 2006; accepted December 1, 2006)

First Department of Internal Medicine, *Department of Pharmacology, Fukushima Medical University, Fukushima, Japan

Mailing address: Yukio Maruyama, MD, First Department of Internal Medicine, Fukushima Medical University, 1 Hikarigaoka, Fukushima 960-1295, Japan. E-mail: maruyama@fmu.ac.jp period $!^{10}$ Our preliminary study using this LVH model revealed that polymorphic VT was induced by programmed electrical stimulation, as shown by Coste et al in their clinical report!1 Thus, the objectives of this study were first, to quantify the vulnerability of VT to programmed extrastimuli, and the changes it undergoes during the development of hypertensive LVH; second, to clarify how this vulnerability of VT relates to arrhythmogenic substrates (ie, conduction disturbances defined by a decrease in conduction velocity and an increase in the fractionation of QRS complex, and repolarization abnormalities defined by increases in the absolute refractory period and monophasic action potential (MAP) duration); and third, to examine how cellular electrophysiological remodeling relates to conduction and repolarization abnormalities of the whole heart during the progress of LVH. For these purposes, we performed programmed electrical stimulation to induce VT at 3 different observation periods in Dahl salt-sensitive (S) rats and -resistant (R) rats, and compared the vulnerability to VT with the conduction velocity and the degree of inhomogeneous conduction evaluated by wavelet analysis, ${ }^{12,13}$ duration of MAP ${ }^{14}$ and absolute refractory period. In addition, myocytes were isolated from the same hearts, and patch-clamp analysis was performed for evaluation of resting membrane potential (RMP) and action potential (AP), as well as sodium ( $\mathrm{INa}$ ), calcium (ICa), and transient outward potassium currents (Ito). 


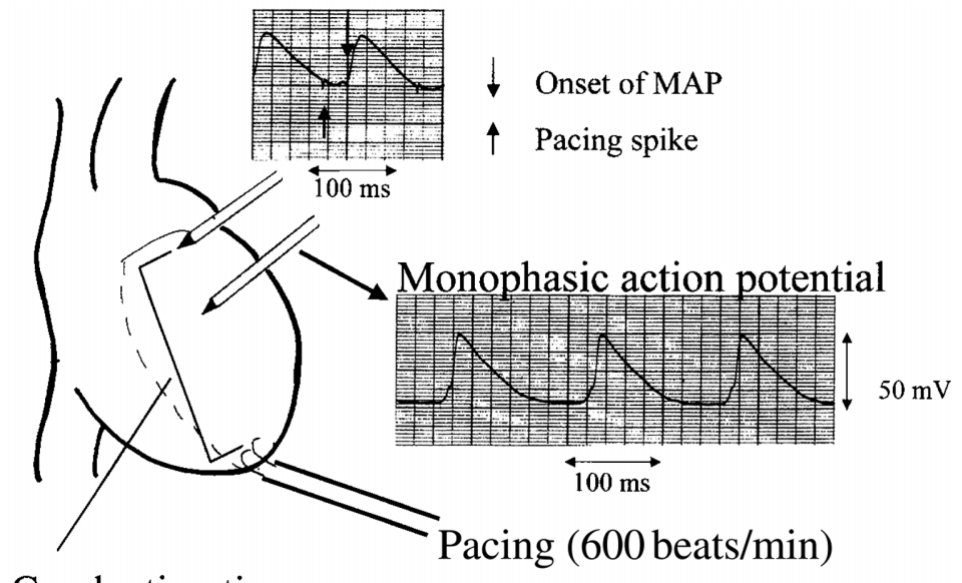

Conduction time
Fig 1. Schematic representation of the recording of the monophasic action potential (MAP). MAP was obtained from the central region of the left ventricular anterior wall. The time from the pacing spike (apical pacing rate: 600 beats $/ \mathrm{min}$ ) to the onset of upstroke of MAP recorded from the base of the left ventricle was defined as the conduction time.

\section{Methods}

This study conformed to the Fukushima Medical University Guidelines for Animal Experiments, the Japanese Government Animal Protection and Management Law (No. 115), and the Guide for the Care and Use of Laboratory Animals (NIH Publication No. 85-23, revised 1996).

\section{Animal Preparation}

Six-week-old male $S$ rats $(n=21)$ and $R$ rats $(n=21)$ (Life Science Center, Japan) were used in this study. They were caged individually in a constant environment (room temperature, $23 \pm 1.5^{\circ} \mathrm{C}$; humidity, $55 \pm 5 \%$ ) and were allowed free access to a high-salt diet $(\mathrm{NaCl} 6 \%)$ and water up to 16 weeks of age. Experiments were performed at 3 different ages to investigate arrhythmogenic substrates during progression of hypertrophy: at 9-10 weeks (10W), 11-13 weeks $(13 \mathrm{~W})$ and $14-16$ weeks old $(16 \mathrm{~W}, \mathrm{n}=7$ for each period in both groups).

\section{Experimental Protocol}

On the day of an experiment, we measured the blood pressure of the tail artery and heart rate using an oscillometric method (UR 5000, Ueda, Japan) while the animal was conscious. The rats were then anesthetized with ether followed by intraperitoneal administration of sodium pentobarbital ( $50 \mathrm{mg} / \mathrm{kg}$ body weight). After anesthesia, hair was shaved in the precordial area for 2-dimensional echocardiography (DE), and on both sides of the chest, the back, head and sacral area for electrocardiogram (ECG) recording. After body surface ECG were recorded for wavelet analysis, 2-DE was performed with the rats supine. The right carotid artery was then cannulated (PE50, Intramedic, USA) for monitoring of aortic blood pressure and fluid supplementation. Next, the chest was opened under artificial ventilation using a rodent ventilator (683, Harvard, USA), and an electrophysiological study of the in situ heart was performed. Finally, the heart was isolated with Langendorff's perfusion, and ventricular myocytes were isolated for whole-cell patch-clamp analysis.

\section{Body Surface ECG and Wavelet Analysis}

Three bipolar ECGs (X lead: at left + and right-axillary line in the 5th intercostal space; Y lead: on the thoracic spine + and sternum-at the same level as X lead; and Z lead: on the sacral+and occipital-region) were obtained
$(0.03-10 \mathrm{kHz})$ in electrically shielded cages using a highprecision amplifier (RECTIGRAPH-8K, Sanei, Japan) and electrodes for electroencephalography (SEE101, Necmed, Japan). Data were recorded on a thermal printer (PC216Ax, Sony, Japan) and stored on magnetic tape (DT-120RA, Sony, Japan) for wavelet analysis. After the experiment, the QRS complex was digitized at $6 \mathrm{kHz}$, and the vector magnitude $\left(\sqrt{\mathrm{X}^{2}+\mathrm{Y}^{2}+\mathrm{Z}^{2}}\right)$ was averaged at approximately $20-40$ consecutive beats as described previously! 13 Wavelet transform was obtained at 40,100,160, 240, 320 and $400 \mathrm{~Hz}$ of the central frequency of the analyzing wavelet, and the area under the wavelet transform at each frequency was calculated as the increase in power with fine fractionation of the QRS complex reflecting inhomogeneous conduction. ${ }^{13}$

\section{Transthoracic 2-DE}

2-DE was performed using an echocardiographic system equipped with a $10-\mathrm{mHz}$ transducer (Hewlett-Packard, SONOS 100CF, USA). Left ventricular (LV) end-diastolic (LVDd) and end-systolic diameters (LVDs), and wall thickness of the interventricular septum (IVS) were measured and shown as average values of 15 consecutive beats. The LV ejection fraction (LVEF) was calculated using the cubic method with the following formula: LVEF $(\%)=$ $\left(\mathrm{LVDd}^{3}-\mathrm{LVDs}^{3}\right) / \mathrm{LVDd}^{3} \times 100$.

\section{MAP and Conduction Velocity}

After the chest was opened, we used hook electrodes for detection of premature ventricular beats while monitoring and recording 3 epicardial orthogonal bipolar ECGs (LV lateral wall to right ventricular free wall; left anterior wall to inferior wall; LV apex to base in the lateral wall). For ventricular pacing, the paired hook electrodes were positioned $2 \mathrm{~mm}$ apart at the LV apex. Wilson's indifferent electrode was constructed from 3 needle electrodes at both upper limbs and the left leg.

After these preparations, the MAP of Wilson's indifferent electrode was obtained by manually pressing the sharpedged $\mathrm{Ag}-\mathrm{AgCl}$ electrodes on the epicardium in the center of the LV anterior wall. The duration of the MAP was measured at the level of $90 \%$ of the maximal amplitude at the base of the LV anterior wall under a ventricular pacing of 600 beats/min (Fig 1); when a ventricular pacing rate of 600 beats/min was impossible, the rate was decreased to 500 beats $/ \mathrm{min}$ and then 400 beats $/ \mathrm{min}$. The time from the pacing spike to MAP onset was defined as the conduction 


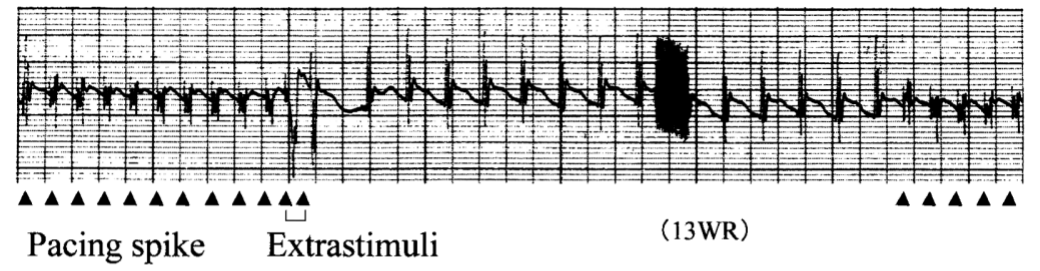

Induction of polymorphic VT

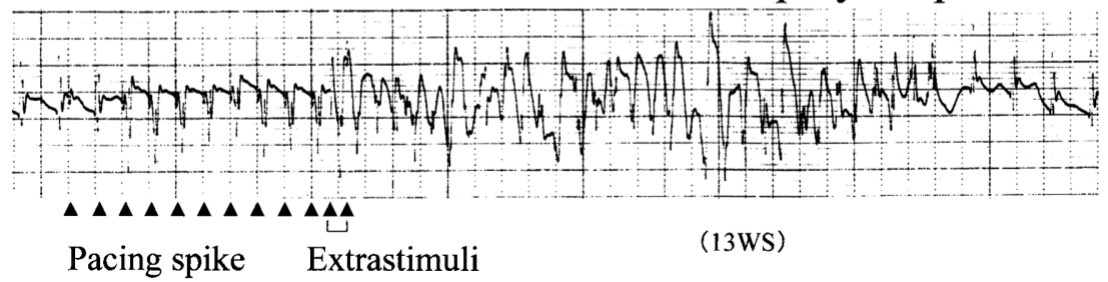

Fig 2. Induction of ventricular tachycardia (VT) by the extrastimulus method. Paired extrastimuli with decreasing coupling interval were added after 10 bouts of pacing (600 beats/min) in $13 \mathrm{~W}$ rats. VT was hardly induced in salt-resistant $(\mathrm{R})$ rats (Top panel). In contrast, polymorphic VT was easily induced in salt-sensitive hypertensive (S) $13 \mathrm{~W}$ rats (Bottom panel).

Table 1 Comparisons of BW, Echocardiographic and Electrophysiological Variables Between S and R Rats

\begin{tabular}{|c|c|c|c|c|c|c|}
\hline & \multicolumn{3}{|c|}{ R rats } & \multicolumn{3}{|c|}{ S rats } \\
\hline & $10 \mathrm{~W}$ & $13 \mathrm{~W}$ & $16 \mathrm{~W}$ & $10 \mathrm{~W}$ & $13 \mathrm{~W}$ & $16 W$ \\
\hline$B W(g)$ & $303 \pm 29$ & $360 \pm 26^{*}$ & $405 \pm 41 * *$ & $312 \pm 31$ & $336 \pm 24$ & $361 \pm 40 *$ \\
\hline$S B P(m m H g)$ & $135 \pm 13$ & $139 \pm 20$ & $146 \pm 16$ & $208 \pm 41^{\# \#}$ & $226 \pm 27^{\# \#}$ & $234 \pm 42^{\# \#}$ \\
\hline$H R$ (beats/min) & $386 \pm 41$ & $418 \pm 57$ & $399 \pm 53$ & $418 \pm 59$ & $428 \pm 58$ & $417 \pm 84$ \\
\hline UCG IVS wall thickness (mm) & $1.1 \pm 0.04$ & $1.1 \pm 0.07$ & $1.2 \pm 0.09$ & $1.5 \pm 0.11^{\#}$ & $1.6 \pm 0.33^{\#}$ & $1.7 \pm 0.52 *$ \\
\hline$E D d(\mathrm{~mm})$ & $5.7 \pm 1.2$ & $6.0 \pm 0.7$ & $5.8 \pm 1.1$ & $6.1 \pm 1.1$ & $5.7 \pm 1.1$ & $5.2 \pm 0.7$ \\
\hline$E F(\%)$ & $95 \pm 3$ & $92 \pm 1$ & $94 \pm 3$ & $91 \pm 5$ & $90 \pm 7$ & $89 \pm 6$ \\
\hline ECG MAP duration $(\mathrm{ms})$ & $66 \pm 4$ & $73 \pm 3$ & $77 \pm 8$ & $81 \pm 11^{\#}$ & $98 \pm 14^{*}, \# \#$ & $114 \pm 15 * * \#$ \\
\hline Conduction velocity $(\mathrm{cm} / \mathrm{s})$ & $76 \pm 12$ & $77 \pm 5$ & $77 \pm 11$ & $73 \pm 4$ & $56 \pm 7^{\# \#}$ & $50 \pm 12 * * \# \#$ \\
\hline
\end{tabular}

$B W$, body weight; S, salt-sensitive; $R$, salt-resistant; SBP, systolic blood pressure; HR, heart rate; UCG, echocardiography; IVS, interventricular septum; $E D d$, end-diastoric dimension; EF, ejection fraction; ECG, electrocardiogram; MAP, monophasic action potential. ${ }^{*} p<0.05$ vs $10 \mathrm{~W},{ }^{* *} p<0.001$ vs $10 \mathrm{~W},{ }^{\#} p<0.05$ vs $R$ rats in same period, ${ }^{\#} p<0.001$ vs $R$ rats in same period.

time (Fig 1). After the distance of the epicardial surface from the pacing site to the MAP recording site was measured using cotton thread, the conduction velocity was calculated as the distance/conduction time $(\mathrm{cm} / \mathrm{s})$.

\section{Measurement of Refractory Period and Induction of VT}

Electrical stimulation was a square wave with a width of $1 \mathrm{~ms}$ at $3.5 \mathrm{~V}$ (Stimulator 3F5I, Sanei, Japan). Extrastimuli were added after 10 round of pacing (S1) at 600 beats $/ \mathrm{min}$. For induction of VT, 2 extrastimuli (S2 and S3) were added after 10 bouts of pacing at 600 beats/min, and coupling intervals that were initially 90 (S1-S2) and $80 \mathrm{~ms}(\mathrm{~S} 2-\mathrm{S} 3)$, as shown in Fig 2, were reduced by $10 \mathrm{~ms}$, respectively, after 10 bouts of pacing; this form of extrastimuli was repeated 10 times. The reduction in the coupling intervals of extrastimuli was repeated until extrastimuli S2 and S3 failed to induce ventricular excitation, after which we increased both $\mathrm{S} 1-\mathrm{S} 2$ and $\mathrm{S} 2-\mathrm{S} 3$ by $2 \mathrm{~ms}$ to find the exact timing needed to induce no ventricular excitation. This period of S1-S2 was defined as the refractory period. VT was defined as equal to or more than 3 consecutive premature ventricular beats (Fig 2). VT vulnerability was defined as the frequency of VT induction during 10 bouts of extrastimuli with certain coupling intervals.

\section{Whole-Cell Patch-Clamp Analysis}

Cell Preparation Single myocytes were enzymatically dissociated from the LV as described previously!5 The excised hearts were perfused on a Langendorff apparatus with the following solutions in sequence: (1) normal Tyrode solution for $2 \mathrm{~min}$ to wash excess blood from the heart; (2) nominally $\mathrm{Ca}^{2+}$-free Tyrode solution for $5 \mathrm{~min}$; (3) enzyme solution containing $0.01 \% \mathrm{WV}^{-1}$ collagenase (Wako, Osaka, Japan), and $0.002 \% \mathrm{WV}^{-1}$ alkaline protease (Nagase, Tokyo, Japan) for 60-80 min; and (4) Kraftbruhe (KB) solution for $3 \mathrm{~min}$. The cells were kept in the KB solution at $4^{\circ} \mathrm{C}$ and were studied within $6 \mathrm{~h}$ of isolation.

Whole-Cell Clamp The whole-cell patch-clamp technique was performed as described previously 16 We generated ramp pulses to induce INa and ICa using a function generator (NF, Tokyo) as described previously! 17 AP was recorded at 10 -s intervals using a $\mathrm{K}^{+}$-pipette solution and Tyrode external solution as described previously 16

Ito was elicited by depolarizing square pulses of 200-ms duration to the voltage range between $-40 \mathrm{mV}$ and $+60 \mathrm{mV}$ with $10 \mathrm{mV}$ steps from the holding potential of $-40 \mathrm{mV}$. The pulse was given every 8 or 10 s. Ito was obtained as a 4aminopyridine-sensitive current by subtracting the current in the presence of 4-aminopyridine from the control currents before applying 4-aminopyridine. The peak for Ito was extrapolated and its amplitude was measured between the peak and a steady state at the end of a 200 -ms pulse. Because currents during the initial $2 \mathrm{~ms}$ after depolarization were capacitative transients, they were omitted. Ito density was obtained by dividing Ito amplitude by the cell capacitance $(\mathrm{Cm})$ in each experiment! 16

Solutions and Drugs The composition of the Tyrode and pipette solutions has been described previously 16 The composition of the modified $\mathrm{KB}$ solution was $70 \mathrm{mmol} / \mathrm{L} \mathrm{KOH}$, $40 \mathrm{mmol} / \mathrm{L} \mathrm{KCl}, 50 \mathrm{mmol} / \mathrm{L} \mathrm{L}$-glutamic acid, $20 \mathrm{mmol} / \mathrm{L}$ 
A

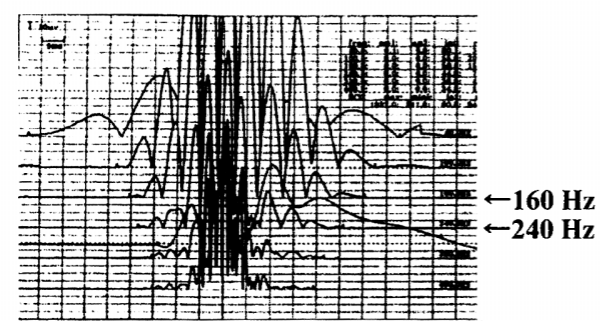

13WS

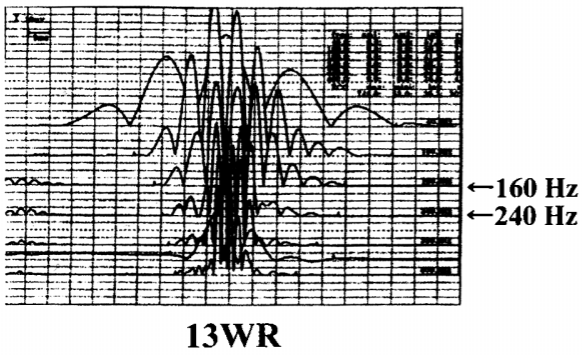

B

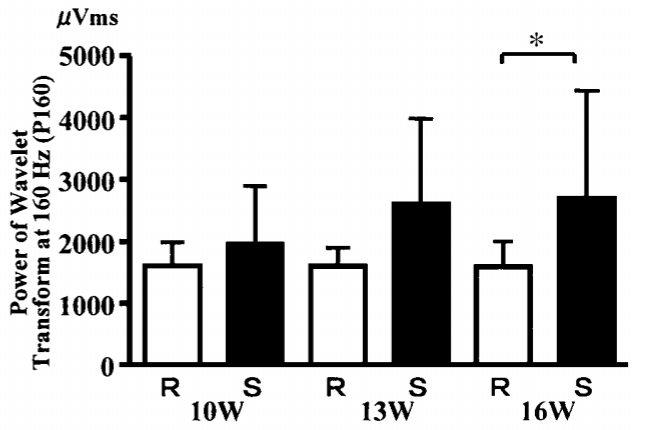

C

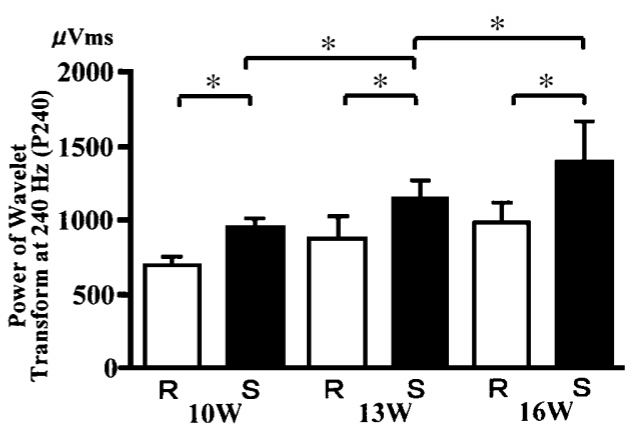

Fig 3. Comparison of wavelet transform of the QRS complex between salt-sensitive hypertensive (S) and salt-resistant normotensive (R) rats. (A) Wavelet transforms of the QRS complex at 40,100,160, 240, 320 and $400 \mathrm{~Hz}$ of the central frequency of the analyzing wavelet were superimposed on the $\mathrm{S}(13 \mathrm{~W}$, Top panel) and $\mathrm{R}(13 \mathrm{~W}$, Bottom panel) rats, showing that the duration and number of peaks increased in $\mathrm{S}$ rats compared with $\mathrm{R}$ rats. The area under the wavelet transform (ie, power) also increased in $\mathrm{S}$ rats compared with $\mathrm{R}$ rats [(B) $160 \mathrm{~Hz}(\mathrm{P} 160),(\mathrm{C}) 240 \mathrm{~Hz}(\mathrm{P} 240)]$. This suggests an increased fine fractionation of the QRS complex, reflecting inhomogeneous conduction. ${ }^{*} \mathrm{p}<0.05$.

taurine, $20 \mathrm{mmol} / \mathrm{L} \mathrm{KH} 2 \mathrm{PO}_{4}, 3 \mathrm{mmol} / \mathrm{L} \mathrm{MgCl} 2,10 \mathrm{mmol} / \mathrm{L}$ glucose, $0.2 \mathrm{mmol} / \mathrm{L}$ ethylene glycol-bis $\beta$-aminoethyl ether)-N,N,N',N'-tetraacetic acid, and $10 \mathrm{mmol} / \mathrm{L}$ HEPES (pH 7.2 adjusted with $\mathrm{KOH}$ ). For recording APs, cells were perfused with Tyrode solution. For the measurements of whole cell Ito $\left(\mathrm{Ca}^{2+}\right.$-insensitive component of Ito), $10 \mathrm{u} \mathrm{mol} / \mathrm{L}$ nifedipine was added to the external solution to block the calcium current, and the pipette solution contained $10 \mathrm{mmol} / \mathrm{L}$ BAPTA to chelate intracellular $\mathrm{Ca}^{2+}$.

\section{Statistical Analysis}

Data are expressed as mean \pm standard deviation. For comparisons among data derived from the 6 groups (ie, $10 \mathrm{~W}, 13 \mathrm{~W}$ and $16 \mathrm{~W}$ in both R and S groups), 1-way analysis of variance was used, followed by Fisher's exact probability test as a post hoc test. Relationships between 2 indices were analyzed by linear regression analysis. A level of $\mathrm{p}<0.05$ was regarded as statistically significant.

\section{Results}

One $\mathrm{S}$ rat died suddenly 16 weeks before the experiment. Therefore, $6 \mathrm{~S}$ rats were examined at $16 \mathrm{~W}$. Spontaneous ventricular premature beats were not observed in any rats during the approximately $60 \mathrm{~min}$ of ECG monitoring (ie, during recording of body surface ECG and the electrophysiological study after thoracotomy). As shown in Table 1, body weight and heart rate in the conscious state did not differ between the 2 groups of rats in each period.

\section{Blood Pressure and LVH}

Systolic blood pressure was much higher in $\mathrm{S}$ rats than in $\mathrm{R}$ rats in all 3 periods, and tended to increase from $10 \mathrm{~W}$ to $16 \mathrm{~W}$. The thickness of the IVS in S rats was greater than

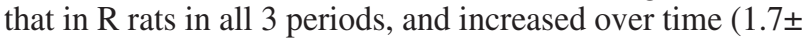
0.52 at $16 \mathrm{~W}$ vs $1.5 \pm 0.11$ at $10 \mathrm{~W}$ in $\mathrm{S}$ rats, $\mathrm{p}<0.05$ ). On the other hand, neither LVEF nor LVDd differed between the 2 groups in all 3 periods or among all 3 periods in each group (Table 1).

\section{Wavelet Analysis of Body Surface ECG}

Fig 3A shows the power profile of wavelet transform at each frequency. The power at 160 and $240 \mathrm{~Hz}$, but not at other frequencies, tended to be different between $\mathrm{R}$ and $\mathrm{S}$ rats in all 3 periods. At $160 \mathrm{~Hz}$, the comparative power in $\mathrm{S}$ rats significantly increased only at $16 \mathrm{~W}$ (Fig 3B). The power of wavelet transform at $240 \mathrm{~Hz}$ increased over time in both $R$ and $S$ rats $(p<0.05$ in $S$, but not in $R$ ), and there were significant differences in the power between $\mathrm{R}$ and $\mathrm{S}$ rats in all 3 periods as shown in Fig $3 \mathrm{C}(\mathrm{p}<0.05$, each).

\section{Electrophysiological Study in the In Vivo Heart}

Systolic blood pressure, wall thickness and MAP duration (S: $81 \pm 11$ vs R: $66 \pm 4 \mathrm{~ms}, \mathrm{p}<0.05$ ) increased at $10 \mathrm{~W}$ in $S$ rats (Table 1). MAP duration in $S$ rats was longer than that in $\mathrm{R}$ rats in all 3 periods ( $\mathrm{p}<0.05$, each), and increased over time (Table 1, Fig 4A). On the other hand, conduction velocity did not decrease at $10 \mathrm{~W}$, but rather declined at $13 \mathrm{~W}$ and $16 \mathrm{~W}$ in S rats compared with R rats. VT was not induced at either $10 \mathrm{~W}$ or $13 \mathrm{~W}$ in $\mathrm{R}$ rats, whereas $\mathrm{R}$ rats at $16 \mathrm{~W}$ showed monomorphic VT at low frequency, but not polymorphic VT, in response to extrastimuli (Fig 4C). Importantly, polymorphic VT was frequently induced at $13 \mathrm{~W}$ and 16W in S rats, but not at 10W (Fig 4C). VT induction among 10 bouts of extrastimuli occurred most frequently at intervals of $\mathrm{S} 1-\mathrm{S} 2$ of $83 \pm 18 \mathrm{~ms}$ at $13 \mathrm{~W}$ and $94 \pm 16 \mathrm{~ms}$ at 

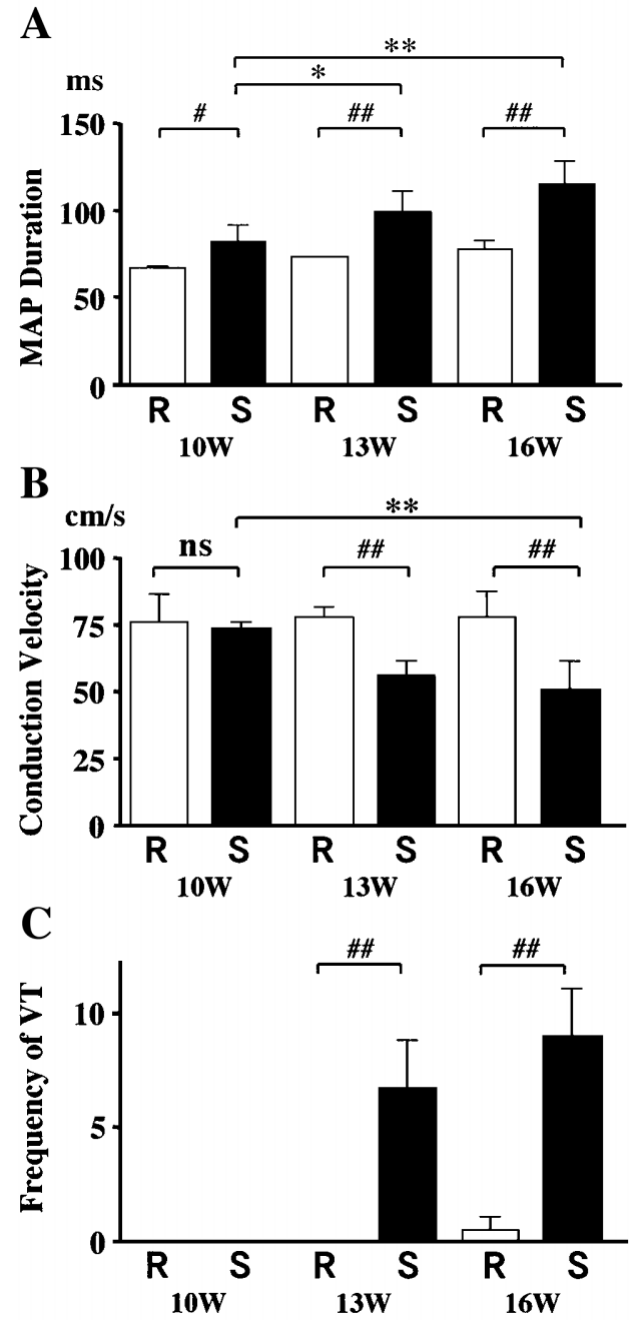

Fig 4. Comparisons of monophasic action potential (MAP) (A), conduction velocity (B), and inducibility of ventricular tachycardia (VT) (C) between salt-sensitive hypertensive (S) and salt-resistant normotensive (R) rats among the 3 time periods. The duration of MAP increased at 10 weeks, and increased over time in $\mathrm{S}$ rats. On the other hand, conduction velocity did not decrease in $\mathrm{S}$ rats at 10 weeks, but decreased in 13 and 16W S rats. VT was not induced at 10 weeks in either $\mathrm{R}$ or $\mathrm{S}$ rats, but was easily induced in hypertensive rats after 13 weeks. ${ }^{*} \mathrm{p}<0.05$ vs $10 \mathrm{~W},{ }^{*} \mathrm{p}<0.001$ vs $10 \mathrm{~W},{ }^{\#} \mathrm{p}<0.05$ vs $\mathrm{R}$ rats in same period, ${ }^{\#} \mathrm{p}<0.001$ vs $\mathrm{R}$ rats in same period.

$16 \mathrm{~W}$ in S rats. Although all these VT inductions were associated with profound hypotension, they spontaneously recovered to sinus rhythm without exception (Fig 2).
Whole-Cell Patch-Clamp Analysis

Table 2 shows the results of whole-cell patch-clamp analysis. RMP and INa did not change over time in $\mathrm{S}$ rats (ie, there were no differences in RMP and INa between the 2 groups in all 3 periods (Table 2), whereas the AP duration at $600 \mathrm{beats} / \mathrm{min}$ of stimulation frequency significantly increased in $\mathrm{S}$ rats at $13 \mathrm{~W}$ and $16 \mathrm{~W}$ ). Ito at $20 \mathrm{mV}$ did not change at $10 \mathrm{~W}$ between $\mathrm{S}$ and $\mathrm{R}$ rats, whereas it decreased over time in $\mathrm{S}$ rats, with a significant reduction at $16 \mathrm{~W}$ compared with $10 \mathrm{~W}(\mathrm{p}<0.05)$. On the contrary, the Ica tended to increase over time in $\mathrm{S}$ rats, but without reaching statistical significance.

\section{Relationships Among the Electrophysiological Variables}

Using data from both $\mathrm{R}$ and $\mathrm{S}$ rats at $10 \mathrm{~W}, 13 \mathrm{~W}$ and $16 \mathrm{~W}$, correlations between VT inducibility and conduction velocity, inducibility of VT and MAP duration, the power of wavelet transform and VT vulnerability, and power of wavelet transform and conduction velocity, were investigated. The inducibility of VT correlated negatively with conduction velocity, indicating that a slow conduction velocity induced VT vulnerability (Fig 5A, r $=-0.83$, p $<$ $0.001)$. The frequency of VT correlated positively with MAP duration (Fig 5B, r=0.69, p<0.001). In addition, there was a significant correlation between conduction velocity and MAP duration ( $\mathrm{r}=-0.64, \mathrm{p}<0.01$, data not shown).

The power of wavelet transform, which indicates the degree of inhomogeneous conduction, correlated inversely with conduction velocity (Fig 6A, 240 Hz (P240): r=-0.69, $\mathrm{p}<0.01$ ) and positively with VT vulnerability (Fig 6B, $240 \mathrm{~Hz}(\mathrm{P} 240)$ : $r=0.66, \mathrm{p}<0.01)$. AP duration obtained by the patch-clamp method correlated positively with MAP duration $(\mathrm{r}=0.69, \mathrm{p}<0.01)$.

\section{Discussion}

This is the first study to comprehensively evaluate the linkage between various arrhythmogenic substrates and vulnerability to VT in hypertensive LVH. Despite the absence of a spontaneous ventricular premature beat in $\mathrm{S}$ rats, polymorphic VT was easily induced by paired extrastimuli, which suggests that arrhythmogenic substrates sufficient to sustain reentrant VT are latently formed, even in compensatory LVH, without enhancement of abnormal impulse formation. In addition, this electrophysiological remodeling consistently progresses, even though the degree of LVH does not significantly increase, as shown from $10 \mathrm{~W}$ to $13 \mathrm{~W}$ in this study. The present study results suggest that not only repolarization abnormalities (ie, increases in MAP and AP duration), but also conduction disturbances (ie, slow conduction velocity) play important roles in the induction of VT in hypertensive LVH, whereas repolariza-

Table 2 Comparison of Cellular Electrophysiological Findings of S and R Rats

\begin{tabular}{|c|c|c|c|c|c|c|}
\hline & \multicolumn{3}{|c|}{$R$ rats } & \multicolumn{3}{|c|}{$S$ rats } \\
\hline & $10 \mathrm{~W}$ & $13 W$ & $16 \mathrm{~W}$ & $10 W$ & $13 W$ & $16 \mathrm{~W}$ \\
\hline$R M P(m V)$ & $-64 \pm 3$ & $-67 \pm 8$ & $-64 \pm 8$ & $-60 \pm 6$ & $-62 \pm 7$ & $-62 \pm 6$ \\
\hline AP duration (ms) & $71 \pm 21$ & $69 \pm 40$ & $67 \pm 12$ & $83 \pm 15$ & $127 \pm 30 * * \# \#$ & $139 \pm 51 * * \# \#$ \\
\hline Na current (nA) & $1.45 \pm 0.21$ & $1.99 \pm 0.44$ & $1.84 \pm 0.58$ & $1.79 \pm 0.35$ & $2.02 \pm 0.76$ & $2.15 \pm 0.80$ \\
\hline Ca current (nA) & $0.48 \pm 0.33$ & $0.47 \pm 0.17$ & $0.46 \pm 0.21$ & $0.49 \pm 0.42$ & $0.70 \pm 0.51$ & $0.70 \pm 0.33$ \\
\hline$I_{t o 20}(p A / p F)$ & $4.44 \pm 1.30$ & $4.56 \pm 1.20$ & $4.28 \pm 0.16$ & $5.02 \pm 1.53$ & $3.47 \pm 2.09$ & $2.08 \pm 1.04^{*, \#}$ \\
\hline
\end{tabular}

$R M P$, resting membrane potential; AP, action potenital; Ito20, transient outward current in $20 \mathrm{mV}$. Other abbreviations see in Table 1. ${ }^{*} p<0.05$ and $* * p<0.01$ vs $10 \mathrm{~W}$ in same $R$ or $S$ rat group; ${ }^{\#} p<0.05$ and ${ }^{\# \#} p<0.01$ vs $R$ rat at in same period. 
A
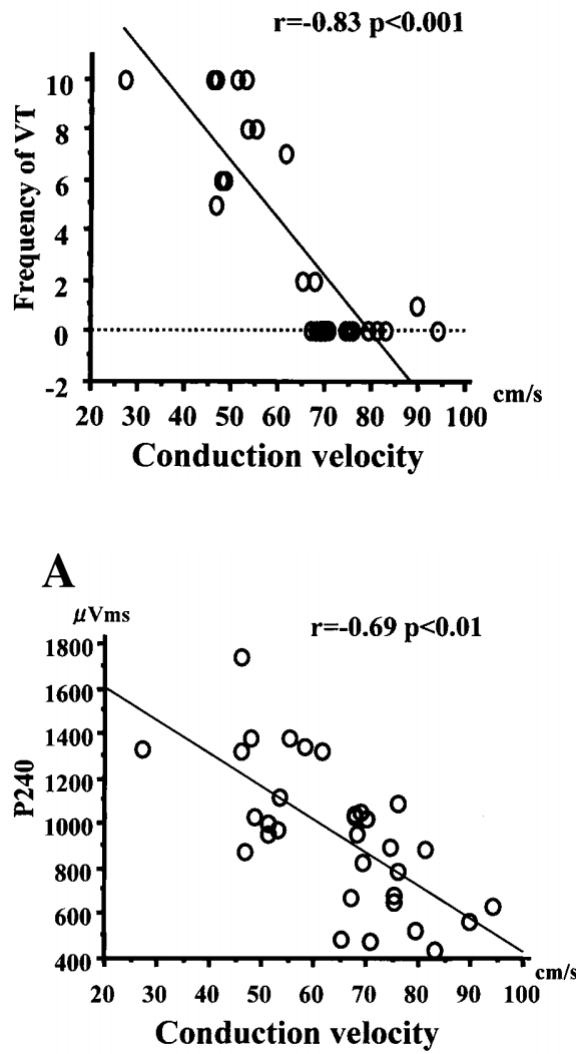

B

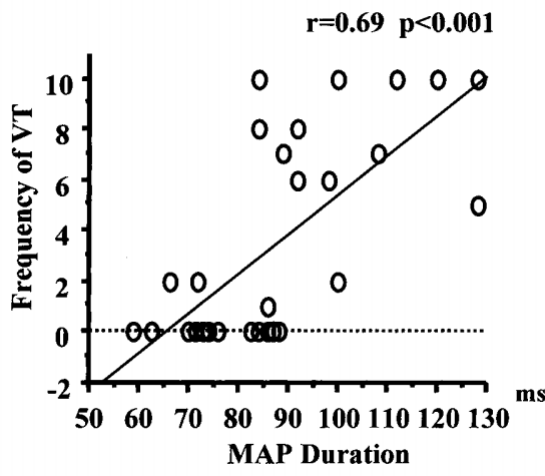

B

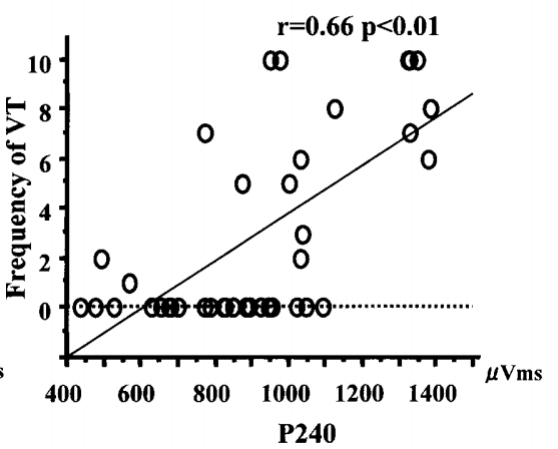

Fig 5. Relationships between inducibility of ventricular tachycardia (VT) and conduction velocity (A) or duration of monophasic action potential (MAP) (B) were obtained using data from both $\mathrm{R}$ and $\mathrm{S}$ rats in $10 \mathrm{~W}$, $13 \mathrm{~W}$ and $16 \mathrm{~W}$. Both conduction velocity $(\mathrm{r}=-0.83, \mathrm{p}<0.001)$ and duration of MAP $(r=0.69, p<0.001)$ were related to the inducibility of VT. S, salt-sensitive hypertensive; $\mathrm{R}$, salt-resistant normotensive.

Fig 6. Relationships between power at $240 \mathrm{~Hz}$ (P240) and conduction velocity (A), inducibility of ventricular tachycardia (VT) and power of wavelet transform (B) were obtained using all data from $\mathrm{R}$ and $\mathrm{S}$ rats. There was a significant correlation between the power of wavelet transform at $240 \mathrm{~Hz}$ and conduction velocity $(\mathrm{r}=-0.69, \mathrm{p}<0.01 ; \mathrm{A})$. Moreover, a significant correlation $(\mathrm{r}=0.66$, $\mathrm{p}<0.01$; B) was found between inducibility of VT and power at $240 \mathrm{~Hz}$. S, salt-sensitive hypertensive; R, salt-resistant normotensive.

tion abnormalities have been shown to be a major cause of polymorphic VT and ventricular fibrillation in hypertrophic cardiomyopathy ${ }^{18}$ In addition, time-frequency analysis using a wavelet transform for the QRS complex of bodysurface ECG may be a clinically useful method for detecting conduction disturbances that increase VT vulnerability in hypertensive $\mathrm{LVH}{ }^{19}$

In terms of determining conduction velocity, the limitation of this study is that we defined conduction velocity as the time from the pacing spike to the MAP onset between the center of LV anterior wall and apex, although recent mapping analysis can determine it accurately. 0,21 In this study, we found that conduction velocity diminished at $13 \mathrm{~W}$ and $16 \mathrm{~W}$, though it was not reduced at $10 \mathrm{~W}$. Previous studies have shown that conduction velocity is somewhat enhanced early in the hypertrophic process, and that this is related to decreased intercellular junctional impedance and enhanced connexin expression.2 ${ }^{2}$ Thereafter, in the process of LVH, conduction velocity decreases with an increase in gap junctional resistance6 In the present study, RMP determining cellular excitability and INa determining the velocity of AP upstroke did not change in rats with $\mathrm{LVH}$, as in previous studies? 23,24 Therefore, the decrease in conduction velocity we observed may relate to cell-to-cell conduction disturbances, presumably because of an increase in gap junctional resistance 25 As previously reported ${ }^{26}$ cardiac interstitial fibrosis may also play a role in conduction disturbances.

The impairment of an intercellular connection can produce an inhomogeneous slow conduction and disruption of the excitation wavefront because of regional conduction blocking in the hypertrophied LV, which results in fine fractionations of the QRS complex of ECG. Wavelet analy- sis is a time-scale technique that estimates the frequency content of a signal as a function of time ${ }^{2}$ It has been reported that highly fractionated local right ventricular electrograms in response to premature stimuli can identify hypertrophic cardiomyopathy patients at risk for sudden cardiac death. ${ }^{27}$ Our results suggest that the fractionation study using wavelet analysis may be a useful non-invasive tool for risk stratification of fatal ventricular arrhythmia in hypertensive LVH.

The increase in AP duration in hypertrophied hearts has been a consistent finding? The duration of AP is primarily responsible for the time course of repolarization of the heart; prolongation of the AP produces delays in cardiac repolarization. It was estimated using computer simulation that the size of a meandering pattern increases, and the degree of VT polymorphism amplified, when AP duration is increased28 The results of the present study support this concept from the experimental point of view. If the electronic interaction between cells is impaired because of an increase in gap junctional resistance, as mentioned earlier, intrinsic differences in AP duration would be revealed, and the enhanced dispersion of repolarization would facilitate reentrant VT 29 In addition, inhomogeneous conduction may further increase the dispersion of repolarization.

The reduction in the current density of Ito is the most consistent ionic current change in cardiac hypertrophy and failure 30 Notable exceptions have been found in studies of compensated pressure overload hypertrophy, which were associated with either no change ${ }^{31}$ or an increase in Ito density 32 This discrepancy in our results seems to be explained by the duration of pressure overload (Tables 1,2). The tendency toward an increase in ICa may also contribute to the prolongation of AP duration, which also seems to depend 
on the duration of pressure overload.

Although it was also a study limitation to quantitatively estimate the relative role of each arrhythmogenic mechanism in a VT attack in this study, our results emphasize that arrhythmogenic substrates that facilitate reentrant VT would latently progress even in compensatory pressure overload $\mathrm{LVH}$, without any manifestation of spontaneous ventricular premature beats, a finding that suggests the usefulness of the fractionation study in predicting the risks of fatal ventricular tachyarrhythmia.

\section{References}

1. Kannel WB, Schatzkin A. Sudden death: Lessons from subsets in subpopulation studies. J Am Coll Cardiol 1985; 5: 141B-149B.

2. Levy D, Anderson KM, Savage DD, Balkus SA, Kannel WB, Castelli WP. Risk of ventricular arrhythmias in left ventricular hypertrophy: The Framingham Heart Study. Am J Cardiol 1987; 60: $560-565$.

3. McLenachan JM, Henderson E, Morris KI, Dargie HJ. Ventricular arrhythmias in patients with hypertensive left ventricular hypertrophy. N Engl J Med 1987; 317: 787-792.

4. Aronson RS. Afterpotentials and triggered activity in hypertrophied myocardium from rats with renal hypertension. Circ Res 1981; 48: $720-727$.

5. Moalic J, Charlemagne D, Mansier P, Chevalier B, Swynghedaum B. Cardiac hypertrophy and failure -a disease of adaptation: Modifications of membrane proteins provide a molecular basis for arrhythmogenicity. Circulation 1993; 87: IV21 -IV26.

6. Cooklin M, Wallis WR, Sheridan D, Fry CH. Changes in cell-to-cell electrical coupling associated with left ventricular hypertrophy. Circ Res 1997; 80: 765-771.

7. Peters NS. Gap junctions and clinical cardiology: From molecular biology to molecular medicine. Eur Heart J 1997; 18: 1697-1702.

8. McIntyre H, Fry $\mathrm{CH}$. Abnormal action potential conduction in isolated human hypertrophied left ventricular myocardium. $J$ Cardiovasc Electrophysiol 1997; 8: 887-894.

9. Bryant SM, Shipsey SJ, Hart G. Regional differences in electrical and mechanical properties of myocytes from guinea-pig hearts with mild left ventricular hypertrophy. Cardiovasc Res 1997; 35: $315-$ 323.

10. Inoko M, Kihara Y, Morii I, Fijiwara H, Sasayama S. Transition from compensatory hypertrophy to dilated, failing left ventricles in Dahl salt-sensitive rats. Am J Physiol 1994; 267: H2471-H2482.

11. Coste P, Clementry J, Besse P, Bricaud H. Left ventricular hypertrophy and ventricular dysrhythmic risk in hypertensive patients: Evaluation by programmed electrical stimulation. J Hypertension 1988; 4: S116-S118.

12. Crowe JA, Gibson NM, Woolfson MS, Somekh MG. Wavelet transform as a potential tool for ECG analysis and compression. J Biomed Eng 1992; 14: 268-272.

13. Maehara K, Kokubun T, Awano N, Taira K, Ono M, Furukawa T, et al. Detection of abnormal high-frequency components in the QRS complex by the wavelet transform in patients with idiopathic dilated cardiomyopathy. Jpn Circ J 1999; 63: 25-32.

14. Franz MR, Lee HC. Relation between repolarization and refractoriness in the human ventricle: Cycle length dependence and effect of procainamide. J Am Coll Cardiol 1992; 19: 614-618.
15. Yazawa K, Kaibara M, Ohara M, Kameyama M. An improved method for isolating cardiac myocytes useful for patch clamp studies. Jpn J Physiol 1990; 40: 157-163.

16. Kimura J, Kawahara M, Sakai E, Yatabe J, Nakanishi H. Effects of a novel cardioprotective drug, JTV-519, on membrane currents of guinea pig ventricular myocytes. Jpn J Pharmacol 1999; 79: $275-$ 281.

17. Hinata M, Yamamura H, Li L, Watanabe $\mathrm{Y}$, Watano T, Imaizumi Y, et al. Stoichiometry of $\mathrm{Na}^{+}-\mathrm{Ca}^{2+}$ exchange is 3:1 in guinea-pig ventricular myocytes. J Physiol 2002; 545: 453-461.

18. Barletta G, Lazzeri C, Franchi F, Del Bene R, Michelucci A. Hypertrophic cardiomyopathy: Electrical abnormalities detected by the extended-length ECG and their relation to syncope. Int J Cardiol 2004; 97: 43-48.

19. Takayama H, Yodogawa K, Katoh T, Takano T. Evaluation of arrhythmogenic substrate in patients with hypertrophic cardiomyopathy using wavelet transform analysis. Circ J 2006; 70: 69-74.

20. Ciaccio EJ. Localization of the slow conduction zone during reentrant ventricular tachycardia. Circulation 2000; 102: 464-469.

21. van Rijen HVM, van Veen TAB, van Kempen MJA, WilmsSchopman FJG, Potse M, Krueger O, et al. Impaired conduction in the bundle branches of mouse hearts lacking the gap junction protein connexin40. Circulation 2001; 103: 1591-1598.

22. Bastide B, Neyses L, Ganten D, Paul M, Willecke K, Traub O. Gap junction protein connexin 40 is preferentially expressed in vascular endothelium and conductive bundles of rat myocardium and is increased under hypertensive conditions. Circ Res 1993; 73: 1138 1149 .

23. Kaufmann RL, Homburger H, Wirth H. Disorder in excitation-contraction coupling of cardiac muscle from cats with experimentally produced right ventricular hypertrophy. Circ Res 1971; 28: 346357.

24. Aronson RS, Nordin C. Electrophysiologic properties of hypertrophied myocytes isolated from rats with renal hypertension. Eur Heart J 1984; 5: 339-345.

25. Wilders R, Verheijck EE, Joyner RW, Golod DA, Kumar R, van Ginneken AC, et al. Effects of ischemia on discontinuous action potential conduction in hybrid pairs of ventricular cells. Circulation 1999; 99: $1623-1629$.

26. Wake R, Kim-Mitsuyama S, Izumi Y, Yoshida K, Izumiya Y, Yukimura T, et al. Beneficial effect of candesartan on rat diastolic heart failure. J Pharmacol Sci 2005; 98: 372-379.

27. Saumarez RC, Slade AK, Grace AA, Sadoul N, Camm AJ, McKenna WJ. The significance of paced electrogram fractionation in hypertrophic cardiomyopathy: A prospective study. Circulation 1995; 91: $2762-2768$

28. Starmer CF, Starobin J. Spiral tip movement: The role of the action potential wavelength in polymorphic cardiac arrhythmias. Int $J$ Chaos Bifurcations 1996; 6: 1909-1023.

29. Haverkamp W, Breithardt G, Camm AJ, Janse MJ, Rosen MR, Antzelevitch C, et al. The potential for QT prolongation and proarrhythmia by non-antiarrhythmic drugs: Clinical and regulatory implications: Report on a policy conference of the European Society of Cardiology. Eur Heart J 2000; 21: 1216-1231.

30. Tomaselli GF, Marban E. Electrophysiological remodeling in hypertrophy and heart failure. Cardiovasc Res 1999; 42: 270-283.

31. Brooksby P, Levi AJ, Jones JV. The electrophysiological characteristics of hypertrophied ventricular myocytes from the spontaneously hypertensive rats. J Hypertens 1993; 11: 611-622.

32. Li Q, Keung EC. Effects of myocardial hypertrophy on transient outward current. Am J Physiol 1994; 266: H1738-H1745. 